\title{
Thrombosis of Both Umbilical Arteries of a Female Fetus in a Twin Gestation Causing Fetal Demise
}

\author{
Shubha P. Bhat ${ }^{1, \odot ~ R i t u r u p a ~ P a u l ~}{ }^{1} \quad$ Teerthanath Srinivas $^{1}$ Shipra Sonkusare ${ }^{2} \quad$ Krishna Priya $^{2}$ \\ ${ }^{1}$ Department of Pathology, K.S. Hegde Medical Academy, \\ Nitte (Deemed to be University), Mangalore, Karnataka, India \\ ${ }^{2}$ Department of Obstetrics and Gynecology, K.S. Hegde Medical \\ Academy, Nitte (Deemed to be University), Mangalore, Karnataka, \\ India \\ Address for correspondence Shubha P. Bhat, MD, Department \\ of Pathology, K.S. Hegde Medical Academy, Nitte (Deemed \\ to be University), Mangalore, Karnataka 575018, India \\ (e-mail: bhatshubha_257@rediffmail.com). \\ J Health Allied Sci ${ }^{\mathrm{NU}}: 2021 ; 11: 97-100$
}

\begin{abstract}
Thrombosis of both umbilical arteries is a rare condition, less common than venous thrombosis. They cause a lot of complications during and after delivery. Umbilical

Keywords

- umbilical artery

- thrombosis

- twin gestation

- female fetus

- histopathology artery thrombosis is associated with both maternal predisposing factors and cord abnormalities and is commonly seen in male fetuses. Presenting symptoms are those of intrauterine growth retardation. Ultrasound abdomen and Doppler flow studies are useful in diagnosing this condition. The prognosis is very poor. Histopathological examination is helpful for confirmation. We present a case of both umbilical arteries thrombosis of a female fetus in a twin gestation causing fetal demise.
\end{abstract}

\section{Introduction}

Thrombosis of the umbilical cord vessels is rare. The incidence is $\sim 1$ for every 1300 deliveries, every 1000 perinatal autopsies, and 250 high-risk gestations. Male fetuses are affected more commonly than females, the ratio being $1.6: 1 .{ }^{1}$ Venous thrombosis accounts for most cases (70\%), followed by combined arterial and venous thrombosis (20\%). Arterial thrombosis alone is rare and accounts for $10 \%$ of cases. ${ }^{2}$ It can be associated with maternal conditions such as diabetes, protein $\mathrm{S}$ deficiency of factor $\mathrm{V}$ Leiden mutation, and cord abnormalities such as long or short cord, hypercoiling, true knots, marginal or velamentous insertion, loops around the body or cervical region, and thin cord with less amount of Wharton's jelly. ${ }^{3}$ Intrauterine growth retardation (IUGR) is usually the presenting feature. During the second trimester, the absence of flow signals during color Doppler performed at the fetal bladder level can help detect umbilical artery thrombosis. ${ }^{4}$ This condition calls for early delivery by cesarean section. ${ }^{3}$ Gross and histopathological examinations are essential for the diagnosis of fibrin clot within the lumen. They are associated with complications that are common in arterial thrombosis than venous thrombosis. ${ }^{5}$ The prognosis of umbilical artery thrombosis is poor, attributing to $80 \%$ of stillbirths in babies with cord thrombosis. ${ }^{1}$

Herein, we present a case of thrombosis of both the umbilical arteries identified during pathological examination of umbilical cord following the fetal demise in one of the female fetuses of a 20-year-old primigravida, delivered at 35 weeks of gestation.

\section{Case Report}

A 20-year-old primigravida with twin gestation, at 35 weeks, and 5 days of gestation, came for a routine antenatal check-up. Ultrasound scan of the abdomen and pelvis showed that one of the twins had no cardiac activity, in breech presentation with overlapping of skull bones with pleural effusion and ascites suggestive of intrauterine death. In contrast, the other published online December 2, 2020
DOI https://doi.org/ 10.1055/s-0040-1721527 ISSN 2582-4287.
C 2020. Nitte (Deemed to be University).

This is an open access article published by Thieme under the terms of the Creative Commons Attribution-NonDerivative-NonCommercial-License, permitting copying and reproduction so long as the original work is given appropriate credit. Contents may not be used for commercial purposes, or adapted, remixed, transformed or built upon. (https://creativecommons.org/licenses/by-nc-nd/4.0/).

Thieme Medical and Scientific Publishers Pvt. Ltd. A-12, 2nd Floor, Sector 2, Noida-201301 UP, India 
had a good cardiac activity with a cephalic presentation. The mother had no history of hyperemesis gravidarum, bleeding per vaginum, or exposure to radiation or teratogenic drugs and was neither hypertensive nor diabetic. She was diagnosed with thyrotoxicosis and managed with propylthiouracil during the first 18 weeks of pregnancy and was currently euthyroid. She had complaints of pain abdomen at 31 weeks, for which she was admitted with clinical suspicion of threatened preterm and managed with tocolytics and steroids. The ultrasound abdomen was normal. She improved symptomatically but was advised for a few more days of hospital stay for which the patient refused. All the previous obstetric scans were unremarkable.

The emergency lower segment cesarean section was performed. The first amniotic sac was ruptured from which straw-colored fluid was drained, and a dead female fetus was delivered by breech extraction weighing $2 \mathrm{~kg}$. The baby was macerated with peeled skin and showed a positive Spalding sign. There were no external anomalies. Cord blood could not be obtained. The second amniotic sac was ruptured from which clear and adequate liquor was delivered. A live baby girl was delivered, weighing $2.32 \mathrm{~kg}$. APGAR score at first and fifth minutes was 9/10. Placenta and membranes were delivered in toto and found to be dichorionic and diamniotic with a placental weight of $920 \mathrm{~g}$. Placenta with attached two umbilical cords was submitted for histopathological examination.

The specimen of the placenta measured $21 \times 20.5 \mathrm{cx} 6 \mathrm{~cm}$, weighing $920 \mathrm{~g}$ with attached two umbilical cords (-Fig. 1). The umbilical cord, which belonged to the dead fetus, measured $29 \mathrm{~cm}$ in length and $1.8 \mathrm{~cm}$ in diameter, with central insertion located at a distance of $6.5 \mathrm{~cm}$ from the placental disc margin. No knots or pseudoknots were noted. The outer surface was congested, dark brown, edematous, and hypocoiled. The umbilical cord cut surface showed three vessels with the lumen of both arteries occluded by thrombus for a distance of $7 \mathrm{~cm}$ at the proximal end (-Fig. $\mathbf{2 A}$ and $\mathbf{B}$ ). Histopathology of the proximal end of larger and longer umbilical cord showed an attached thrombus in both the umbilical arteries ( $\mathbf{- F i g . ~ 3 A}$ ), with the tunica media showing scattered neutrophils ( - Fig. 3B). Mesenchymal tissue was edematous. The umbilical vein was unremarkable ( - Fig. $\mathbf{3 C}$ ). Sections studied from dividing membranes showed focal fibrinoid necrosis, neutrophils with denuded amnion, and chorionic epithelium with calcification (-Fig. 4). Fetal surface of the placental disc belonging to stillborn fetus showed coagulation necrosis, extensive intravillous fibrin with calcification, and crowding of villi with increased syncytial knots (-Fig. 5). The maternal surface showed focal areas showing

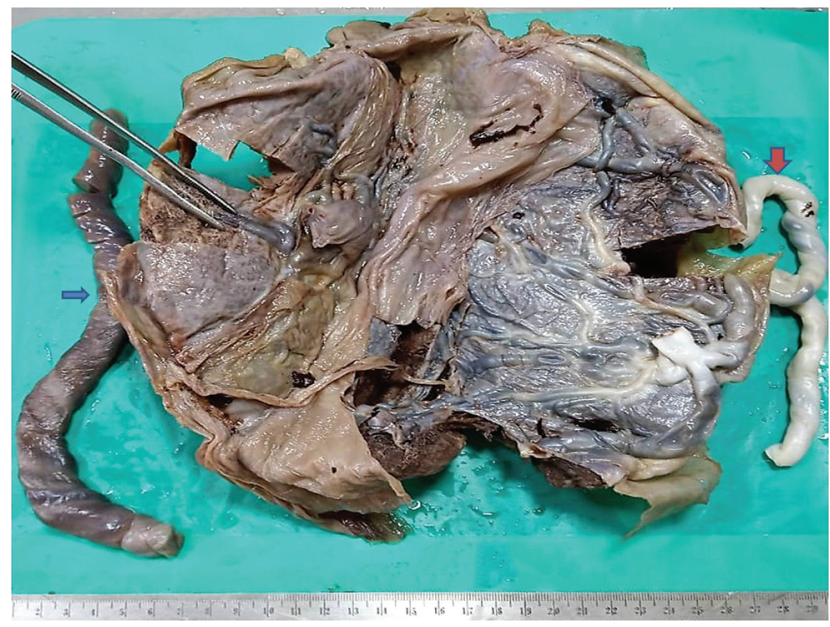

Fig. 1 Gross specimen of the placenta with attached two umbilical cords, one which is dark brown, congested, and edematous (blue arrow) and other normal umbilical cord (red arrow).
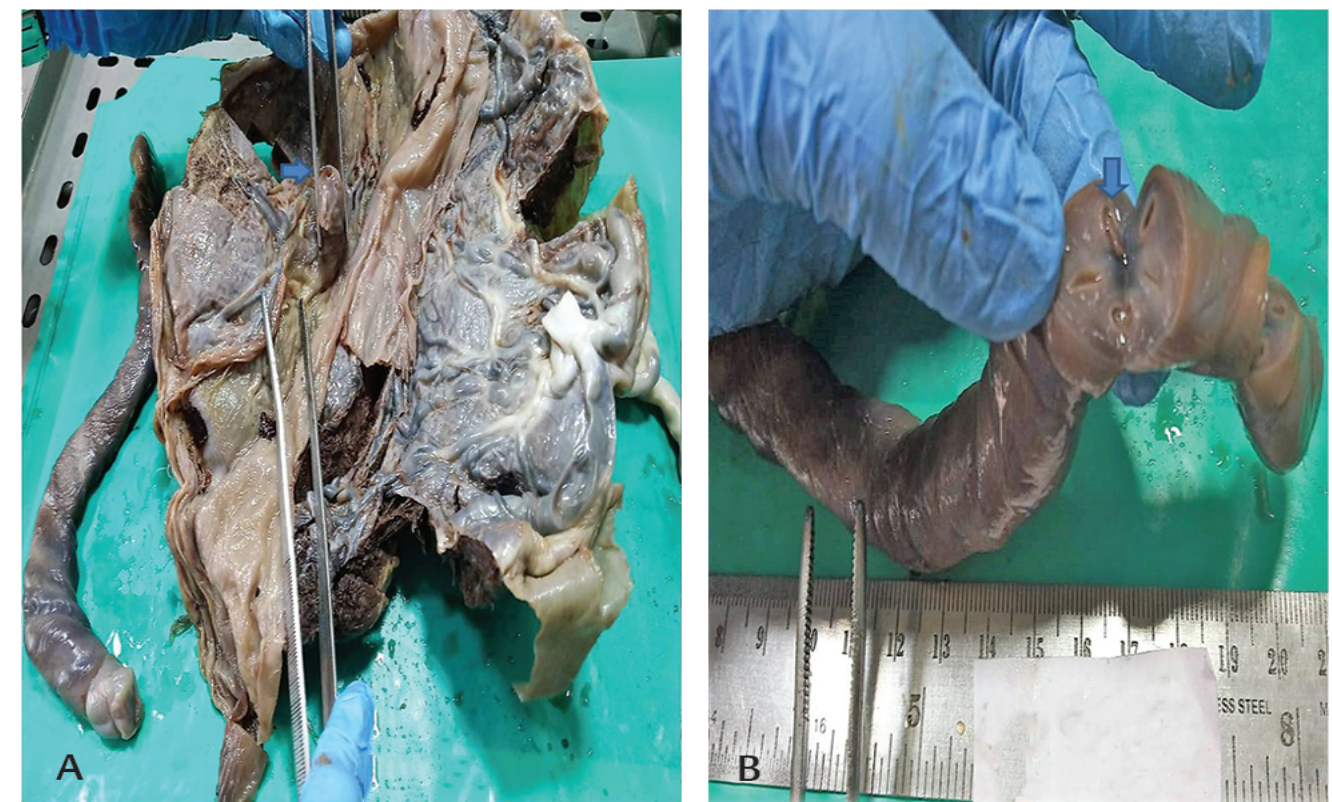

Fig. 2 (A) Cut surface of umbilical cord showing three vessels with the lumen of both arteries occluded by a thrombus (arrow). (B) Closer view of the same, showing the thrombus (arrow). 

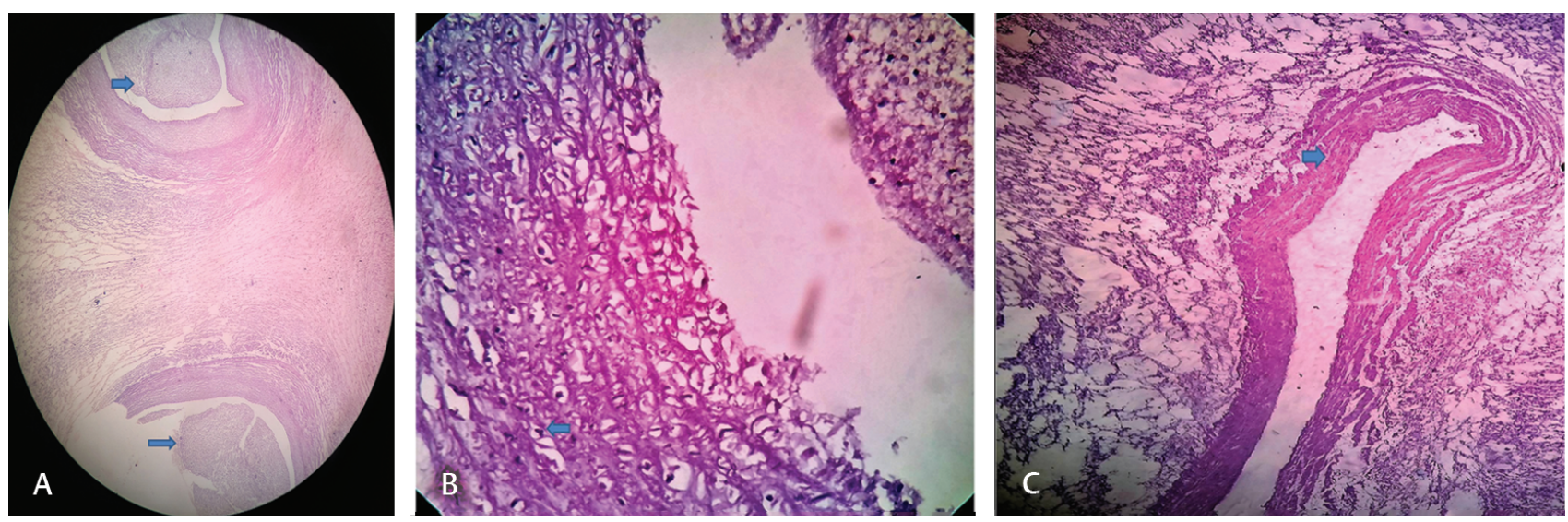

Fig. 3 (A) Microscopy showing an attached thrombus in both umbilical arteries (arrows); (B) microscopy showing neutrophils in the vessel wall (arrow); (C) microscopy of the normal umbilical vein. Hematoxylin and eosin, 10x.

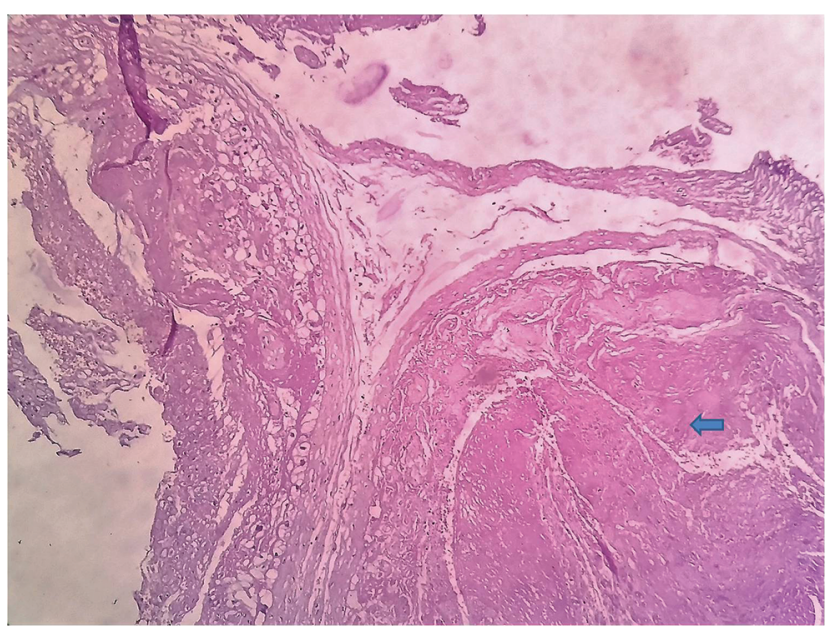

Fig. 4 Microscopy of membranes showing denuded epithelium with fibrinoid necrosis (arrow), hematoxylin and eosin, 10x.

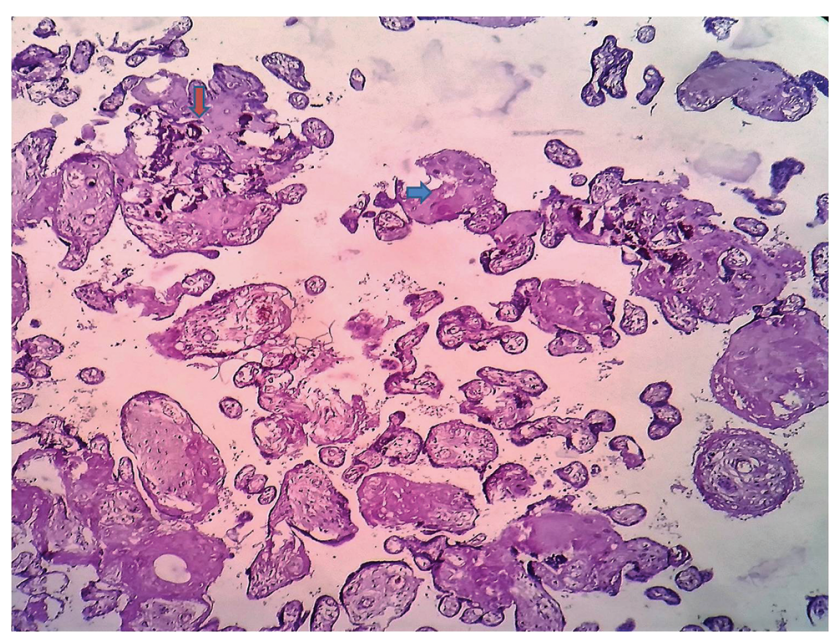

Fig. 5 Microscopy of the fetal surface of the placental disc of the dead baby showing extensive intravillous fibrin (blue arrow) and calcification (red arrow), hematoxylin and eosin, 10x.

calcification of decidual plate with intravillous fibrin. Features were suggestive of thrombosis of both the umbilical arteries with chronic maternovascular malperfusion and funisitis.
The other umbilical cord measured $24 \mathrm{~cm}$ in length and $1 \mathrm{~cm}$ in diameter with marginal insertion. Coiling appeared to be normal. No knots and pseudoknots were noted. The outer surface was pale white. Cut surface showed three vessels with a patent lumen. Membranes appeared translucent. The rupture of membranes was marginal. The dividing membrane was thick and opaque. The fetal surface of the placental disc was unremarkable. The maternal surface showed cotyledons with few pale white areas.

The postoperative period was uneventful. Blood parameters and coagulation profile were within normal limits.

\section{Discussion}

The umbilical cord helps in exchanging nutrition and oxygen between the mother and the baby. It has two arteries that carry deoxygenated blood from fetus and one umbilical vein that carries oxygenated blood to the fetus. Hence, umbilical vein thrombosis can be lethal to the fetus. ${ }^{6}$ Some of the common conditions affecting the umbilical cord are a single umbilical artery, cord prolapse, vasa previa, and umbilical cord knots. Umbilical vessel thrombosis is a rare condition, which can cause fetal hypoxia and, eventually, fetal death. Umbilical vessel thrombosis is around 1.6 times more common in male fetuses. ${ }^{1}$ Thrombosis of the umbilical vein is more common than arterial thrombosis. ${ }^{2}$ In the present case, thrombosis of both umbilical arteries was noted in a female fetus. There has been an association of umbilical vessel thrombosis with maternal conditions such as diabetes, thrombophilic disorders such as protein $\mathrm{S}$ deficiency or factor V Leiden mutation, and cord abnormalities such as the presence of a long cord $(>70 \mathrm{~cm})$ or short cord $(<35 \mathrm{~cm})$, excessive twisting ( $>0.3 \mathrm{~cm} /$ loop), reduced diameter $(<8.0 \mathrm{~mm})$, anomalous placental insertions, and presence of true knots and loops. ${ }^{3}$ Etiopathogenesis of umbilical vessel thrombosis may be explained by the Virchow's triad, which includes hypercoagulability, endothelial injury, and blood stasis. Hypercoagulability may be due to inherited or acquired maternal or fetal thrombophilia. Endothelial injury may be due to funisitis or meconium-induced vascular 
necrosis. Stasis of blood may be caused by mechanical or anatomical obstruction. ${ }^{6}$ In the present case, there was evidence of endothelial damage, causing funisitis of umbilical arteries. There was no history of diabetes mellitus or hypercoagulability in the mother or evidence of umbilical cord anomalies. Symptoms are usually those of IUGR, and umbilical vessels' thrombosis should be suspected if no other cause of IUGR can be determined.

In the present case, the patient had an episode of pain abdomen at 31 weeks, which was suspected to be threatened preterm and was managed accordingly. The ultrasound abdomen was normal. However, to evaluate further, the patient refused advice on further stay at the hospital.

Prenatal diagnosis of umbilical artery thrombosis remains a clinical challenge with minimal literature on umbilical artery thrombosis cases being diagnosed by ultrasound examination. When one of the umbilical arteries is thrombosed, it is usually misdiagnosed to be a single umbilical artery. This can be avoided by comparing it with earlier ultrasound scans. ${ }^{7}$ On color Doppler, "orange grabbed sign" is the characteristic finding seen in umbilical artery thrombosis. The occluded artery and the normal artery are surrounded by the uterine vein, which appears like "an orange grabbed by a hand." The highly curving "C-shaped" vein, which surrounds the arteries, may indicate hypercoiling of the umbilical cord. This can help in the prenatal diagnosis and help in perinatal fetal management. ${ }^{8} 7$ Intrauterine death of one of the twin fetuses was diagnosed on ultrasonography when performed at 35 weeks, 5 days during her routine antenatal visit.

Cord thrombosis is associated with many complications such as intrauterine death, respiratory distress, skull abnormalities, and low birth weight. ${ }^{5}$ Inducing fetal lung maturation by giving steroids after 34 weeks of gestation, early delivery by cesarean section, and monitoring APGAR score can help manage fetuses with umbilical vessel thrombosis. ${ }^{3}$

Grossly, the umbilical cord may be swollen and edematous with identifiable thrombi on the cut surface. Histologically, in contrast to umbilical vein thrombosis, thrombotic umbilical cord arteries show partial necrosis of the vessel wall, with the inner layer being more commonly affected. Since the umbilical arteries lack vasa vasorum, oxygen supply to the intima is provided by the blood flow, and to the outer layer by amniotic fluid, thrombi occluding the lumen can cause necrosis of the inner layer with sparing of the outer layer. ${ }^{2}$ Since umbilical vessels have no vasa vasorum, thrombi are not organized, which leads to necrosis and calcification within the thrombi. ${ }^{4}$ In the present case, thrombus in both the umbilical arteries was evident on the gross and microscopic examination, which may have been due to undetected maternofetal infection (funisitis and chorioamnionitis), causing endothelial injury, as seen by the presence of neutrophilic infiltrate in the arterial wall. Also, there was twin gestation in the present case, resulting in external compression of cord by the other fetus, resulting in the stasis of blood and thrombosis.

Hyrtl's anastomosis, which is the anastomosis of two umbilical arteries, can be seen in $90 \%$ of placentas. This anastomosis can prevent placental hypoxia and infarctions caused by umbilical artery thrombosis. ${ }^{9}$ In the present case, no Hyrtl's anastomosis was found on histological examination. Fetal surface of placental disc belonging to dead fetus showed features of chronic maternovascular malperfusion.

\section{Conclusion}

Umbilical artery thrombosis is a rare condition and has a poor prognosis causing perinatal morbidity and mortality. Usually, it is associated with many predisposing maternal and umbilical cord factors but sometimes remains idiopathic. Prenatal detection of umbilical artery thrombosis by ultrasound scan remains a challenge to the radiologists, although using color Doppler may help diagnose it. There are chances that thrombus may rapidly occur between antenatal visits. Hence, the antenatal visits must be closely monitored in the third trimester, and any abnormalities should be managed as early as possible to avoid adverse pregnancy outcomes.

\section{Conflict of Interest}

None declared.

\section{References}

1 Heifetz SA. Thrombosis of the umbilical cord: analysis of 52 cases and literature review. Pediatr Pathol 1988;8(1):37-54

2 Sato Y, Benirschke K. Umbilical arterial thrombosis with vascular wall necrosis: clinicopathologic findings of 11 cases. Placenta 2006;27(6-7):715-718

3 Oliveira GH, Dias CdeM, Vaz-Oliani DCM, Oliani AH. Intrauterine thrombosis of umbilical artery - case report. Sao Paulo Med J 2016;134(4):355-358

4 Klaritsch P, Haeusler M, Karpf E, Schlembach D, Lang U. Spontaneous intrauterine umbilical artery thrombosis leading to severe fetal growth restriction. Placenta 2008;29(4):374-377

5 Shilling C, Walsh C, Downey P, Mooney E. Umbilical artery thrombosis is a rare but clinically important finding: a series of 7 cases with clinical outcomes. Pediatr Dev Pathol 2014; 17(2):89-93

6 Lutfallah F, Oufkir N, Markou GA, Frimigacci D, Poncelet C. A case of umbilical artery thrombosis in the third trimester of pregnancy. Am J Case Rep 2018;19:72-75

7 Li H, Wu Q, Wei W, Lin X, Zhang X. Umbilical artery thrombosis: two case reports. Medicine (Baltimore) 2019;98(48):e18170

8 Tanaka K, Tanigaki S, Matsushima M, et al. Prenatal diagnosis of umbilical artery thrombosis. Fetal Diagn Ther 2014; 35(2):148-150

9 Avagliano L, Marconi AM, Candiani M, Barbera A, Bulfamante G. Thrombosis of the umbilical vessels revisited. An observational study of 317 consecutive autopsies at a single institution. Hum Pathol 2010;41(7):971-979 